\title{
Decreased TGFBR3/betaglycan expression enhances the metastatic abilities of renal cell carcinoma cells through TGF- $\beta$-dependent and -independent mechanisms
}

\author{
Jun Nishida ${ }^{1} \cdot$ Kohei Miyazono ${ }^{1} \cdot$ Shogo Ehata $\mathbb{1}^{1}$ \\ Received: 1 May 2017 / Revised: 30 November 2017 / Accepted: 1 December 2017 / Published online: 2 February 2018 \\ (c) The Author(s) 2018. This article is published with open access
}

\begin{abstract}
TGF- $\beta$ regulates both the tumor-forming and migratory abilities of various types of cancer cells. However, it is unclear how the loss of TGF- $\beta$ signaling components affects these abilities in clear-cell renal cell carcinoma (ccRCC). In this study, we investigated the role of TGFBR3 (TGF- $\beta$ type III receptor, also known as betaglycan) in ccRCC. Database analysis revealed decreased expression of TGFBR3 in ccRCC tissues, which correlated with poor prognosis in patients. Orthotopic inoculation experiments using immunocompromised mice indicated that low TGFBR3 expression in ccRCC cells enhanced primary tumor formation and lung metastasis. In the presence of TGFBR3, TGF- $\beta 2$ decreased the aldehyde dehydrogenase (ALDH)-positive ccRCC cell population, in which renal cancer-initiating cells are enriched. Loss of TGFBR3 also enhanced cell migration in cell culture and induced expression of several mesenchymal markers in a TGF- $\beta$ independent manner. Increased lamellipodium formation by FAK-PI3K signaling was observed with TGFBR3 downregulation, and this contributed to TGF- $\beta$-independent cell migration in ccRCC cells. Taken together, our findings reveal that loss of TGFBR3 endows ccRCC cells with multiple metastatic abilities through TGF- $\beta$-dependent and independent pathways.
\end{abstract}

\section{Introduction}

Cancer metastasis is a multi-step process that includes growth and migration in primary sites, intravasation, dissemination to distant organs, extravasation, and colonization at the secondary site [1]. To accomplish these sequential steps, it is essential for cancer cells to acquire both tumor-forming and cell-migratory abilities. To explain this process, several models including cancer stem-cell theory have been proposed [2].

Transforming growth factor- $\beta$ (TGF- $\beta$ ) signaling is involved in many biological and pathological processes [3].

Electronic supplementary material The online version of this article (https://doi.org/10.1038/s41388-017-0084-0) contains supplementary material, which is available to authorized users.

Kohei Miyazono

miyazono@m.u-tokyo.ac.jp

1 Department of Molecular Pathology, Graduate School of Medicine, The University of Tokyo, 7-3-1 Hongo, Bunkyo-ku, Tokyo 113-0033, Japan
Three members of TGF- $\beta$, namely, TGF- $\beta 1$, TGF- $\beta 2$, and TGF- $\beta 3$, have been identified in mammals. TGF- $\beta$ signaling is transduced through receptor complexes with dual protein kinase activities, comprises TGF- $\beta$ type II receptor (T $\beta$ RII, encoded by the TGFBR2 gene) dimer and type I receptor (T $\beta R I$, also known as activin receptor-like kinase 5 (ALK-5), encoded by the TGFBRl gene) dimer. TGF- $\beta$ initially binds to T $\beta$ RII, which phosphorylates and activates T $\beta R I$. Activated T $\beta$ RI phosphorylates receptor-regulated Smads (R-Smads), Smad2 and Smad3. R-Smads then bind to the common-partner Smad (co-Smad), Smad4, and translocate into the nucleus to regulate target gene transcription. These regulatory mechanisms are highly modulated by accessory receptor proteins, transcription factors, and transcriptional co-factors, which associate with receptors or Smads. TGF- $\beta$ also activates non-Smad signaling pathways including mitogen-activated protein kinase (MAPK) signaling pathways [4-6].

Although TGF- $\beta$ signaling affects cancer cell phenotypes by regulating tumor-forming and cell-migratory abilities, these effects are bidirectional depending on the stage of cancer progression [7, 8]. During the early stages of cancer 
progression, TGF- $\beta$ has tumor-suppressive roles by inhibiting cell growth or attenuating cancer-initiating cell (CIC) maintenance. Numerous studies have indicated that genes encoding TGF- $\beta$ signaling components, such as TGFBR2 and SMAD4, are frequently mutated or epigenetically silenced in many types of cancer cells [9]. As these alterations contribute to the attenuated TGF- $\beta$ signaling, cancer cells become resistant to the growth inhibitory effects of TGF- $\beta$ during advanced stages. TGF- $\beta$ also induces epithelial-mesenchymal transition (EMT) in cancer cells to promote their invasion and migration [10]. However, it is possible that loss of TGF- $\beta$ signaling components also leads to the attenuation of TGF- $\beta$-mediated EMT [11]. These facts are contradictory to the metastatic properties of cancer cells after TGF- $\beta$ signaling components are mutated or deleted. Thus, alternative pathways, through which cancer cells overcome this event, might be present.

Clear-cell renal cell carcinoma (ccRCC) is the most common subtype of kidney cancer, and metastasizes to various organs including lung, bone, brain, and lymph nodes [12]. ccRCC is also well characterized as highly vascularized cancer. The main cause of vascularization is a disruption in von Hippel-Lindau (VHL) tumor suppressorhypoxia inducible factor (HIF) signaling through genetic or epigenetic mechanisms [13]. VHL-HIF signaling has been reported to positively regulate both tumor-initiating ability and cell-migratory ability in ccRCC cells [14]. It was reported that the absence of VHL results in overexpression of TGF- $\beta 1$, which facilitates angiogenesis in a paracrine manner [15]. A previous study identified the loss of certain TGF- $\beta$ receptors, namely, T $\beta$ RII and TGFBR3 (TGF- $\beta$ type III receptor, also known as betaglycan), during ccRCC progression [16], implying a disruption in TGF- $\beta$ signaling in renal carcinogenesis. However, the detailed function of TGF- $\beta$ signaling in ccRCC cells is poorly understood. Thus, it is necessary to elucidate how the loss of TGF- $\beta$ signaling components affects metastasis in ccRCC cells.

In the present study, we focused on the tumor-suppressive role of TGFBR3 in advanced ccRCC. On the basis of results using an in vivo renal orthotopic tumor model, we hypothesized that the loss of TGFBR3 enhances multiple metastatic abilities of ccRCC cells. Loss of TGFBR3 increased the CIC-enriched, aldehyde dehydrogenase (ALDH)-positive cell population by attenuating TGF$\beta 2$ signaling. Moreover, loss of TGFBR3 enhanced cellmigratory ability through an alternative pathway independent of TGF- $\beta$-T $\beta$ RI signaling. Our findings provide new insight into the acquisition of a metastatic phenotype after the loss of TGF- $\beta$ signaling components in ccRCC cells.

\section{Results}

\section{TGFBR3 acts as a tumor suppressor during CCRCC progression}

To identify aberrant TGF- $\beta$ signaling in ccRCC, altered expression of signaling components such as receptors and Smads was analyzed using The Cancer Genome Atlas (TCGA) data sets for ccRCC (Fig. 1a; Supplementary Figure S1A). Expression of some TGF- $\beta$ signaling components was only slightly different between normal kidney and ccRCC tissues. In contrast, expression of TGFBR3 was lower in stage I ccRCC tissues than that in normal kidney tissues (Fig. 1a). Comparisons between stage I ccRCC and stage III or IV ccRCC indicated that expression of TGFBR3 was also reduced in ccRCC in a clinical stage-dependent manner (Fig. 1a). Further analysis also showed that low $T G F B R 3$ expression was associated with poor prognosis in this data set (Fig. 1b).

To determine whether decreased expression of TGFBR3 only occurs in ccRCC cells, levels of TGFBR3 were compared in many types of cancer cells. Microarray data from the Cancer Cell Line Encyclopedia (CCLE) revealed that median expression of TGFBR3 mRNA was third lowest in RCC cell lines among 24 types of cancer cell lines (Supplementary Figure S1B). Expression of TGFBR3 mRNA was also examined by quantitative real-time reverse transcription-PCR (qRT-PCR). All examined ccRCC cells expressed low levels of TGFBR 3 compared to normal renal cells, HEK 293 (Fig. 1c). In particular, several ccRCC cells, such as OS-RC-2 and 786-O, exhibited extremely diminished TGFBR3 expression. This suggested that TGFBR3 was downregulated in ccRCC cells.

To evaluate the role of TGFBR3 in the progression of ccRCC, we established OS-RC-2 cells overexpressing TGFBR3 (OS-RC-2-TGFBR3) or Caki-1 cells with silenced TGFBR3 expression using two shRNAs (Caki-1shTGFBR3) (Fig. 2a, b). TGFBR3 is expressed as a protein with or without glycosaminoglycan (GAG) chains [17]. As TGFBR3 with GAG chains is observed as a very broad band in SDS-gels, only TGFBR3 without GAG chains (core protein) can be detected by immunoblot analyses. Accordingly, OS-RC-2-TGFBR3 and control Caki-1 cells (Caki-1shNTC) expressed the TGFBR3 core protein with $\sim 110 \mathrm{kDa}$ in their cellular membrane fraction (Fig. 2a, b). The progression of ccRCC was evaluated in vivo using a mouse renal orthotopic tumor model. Herein, ccRCC cells were inoculated into the renal subcapsule of immunocompromised mice, and tumor progression was monitored by bioluminescence imaging. Overexpression of TGFBR3 in OSRC-2 cells significantly reduced primary tumor progression (Fig. 2c) and prolonged survival (Fig. 2d). Conversely, downregulation of TGFBR3 expression in Caki-1 cells 
Fig. 1 TGFBR3 is

downregulated in ccRCC cells and tissues. a Expression of

TGF- $\beta$ receptors in human normal renal tissues and ccRCC tissues was analyzed using the TCGA database (kidney renal clear-cell carcinoma, KIRC). All data were divided into normal $(n=68)$, stage I $(n=223)$, stage II $(n=49)$, stage III $(n=119)$, and stage IV $(n=80)$ ccRCC.

The fragments per kilobase of exon per million mapped sequence reads (FPKM) was calculated for each gene to compensate the number of transcript fragment based on the length of genes and was shown by dot plots $(* * * p<0.001$; oneway ANOVA with Tukey's test). $E N G$ : gene encoding endoglin. b Relationship between TGFBR3 expression and overall survival in ccRCC patients was analyzed using the TCGA database (KIRC) by Kaplan-Meier plot. Samples were divided into either TGFBR3 ${ }^{\text {high }}(n=231)$ or TGFBR $3^{\text {low }}(n=231)(* * p<$ 0.01 ; log-rank test). c Expression of TGFBR3 mRNA was quantified by qRT-PCR analysis. HEK 293 and HK-2 cells were used as normal renal cells. ACHN, Caki-1, OS-RC-2, and $786-\mathrm{O}$ cells were used as ccRCC cells. Data represent mean number of duplicate samples + SD
A

TGFBR1


B


TGFBR2


C



significantly increased primary tumor progression (Fig. 2e). Expression of TGFBR3 mRNA remained to be decreased in the primary tumors of Caki-1-shTGFBR3-xenograted mice (Supplementary Figure S2). Moreover, ex vivo bioluminescence imaging of resected lungs revealed that spontaneous metastatic lung tumors derived from Caki-1shTGFBR3 cells were significantly larger than those from Caki-1-shNTC cells (Fig. 2f). These results suggested that
TGFBR3 acts as a tumor suppressor during ccRCC progression.

\section{TGFBR3 is required for TGF- $\beta 2$-mediated signal transduction in ccRCC cells}

The transmembrane form of TGFBR3 is known as an accessory protein that promotes binding of TGF- $\beta$ to T $\beta R I I$ 
A

OS-RC-2


C



E



B

Caki-1


D



F



to enhance T $\beta R I-d e p e n d e n t$ signal transduction [17]. Thus, the signaling activity of each TGF- $\beta$ in ccRCC cells was determined. Each TGF- $\beta$ was expressed in renal tissue and other tissues known as metastatic sites for ccRCC
(Supplementary Figure S3A). CCLE microarray data revealed that median expression of TGFB2 mRNA in RCC cell lines was fifth highest among 24 types of cancer cell lines, implying that a growth inhibitory, autocrine TGF- 
Fig. 2 Effect of TGFBR3 on primary tumor formation and metastasis in ccRCC. a (Top) Expression of TGFBR3 mRNA in OS-RC-2 cells was quantified by qRT-PCR analysis. Data represent mean number of duplicate samples + SD. (Bottom) Expression of TGFBR3 core protein in OS-RC-2 cells was determined by immunoblotting. b (Top) Expression of TGFBR3 mRNA in Caki-1 cells was quantified by qRTPCR analysis. Data represent mean number of duplicate samples + SD. (Bottom) Expression of TGFBR3 core protein in Caki-1 cells was determined by immunoblotting. TGFBR3 was blotted after immunoprecipitation with anti-TGFBR3 antibody. $\mathbf{c}$ Tumor progression of OSRC-2-GFP and OS-RC-2-TGFBR3 cells was analyzed using a mouse renal orthotopic tumor model 14 days after inoculation. (Left) Primary tumor was visualized by in vivo bioluminescence imaging $(n \geq 5)$. (Right) Overall luminescence in each mouse is converted to base 10 logarithmic scale and shown by dot plot. Basal overall luminescence is set to 1 . Mean number is also indicated (**p $<0.01$; Welch's $t$ test). d Relationship between TGFBR3 expression and survival in tumorbearing mice from $\mathbf{c}$ was analyzed by Kaplan-Meier plot $\left({ }^{*} p<0.05\right.$; log-rank test). e Tumor progression of Caki-1-shNTC and Caki-1shTGFBR3 cells was analyzed using a mouse renal orthotopic tumor model 28 days after inoculation. (Left) Primary tumors were detected by in vivo bioluminescence imaging $(n=16)$. (Right) Overall luminescence in each mouse is converted to base 10 logarithmic scale and shown by dot plot. Mean number is also indicated $(* p<0.05, * * p<$ 0.01; one-way ANOVA and Tukey's test). f Spontaneous metastatic lung tumor formation from mice in e was analyzed 42 days or 43 days after inoculation. (Left) Metastatic lung tumors were detected by ex vivo bioluminescence imaging $(n=16)$. (Right) Overall luminescence in each lung is converted to base 10 logarithmic scale and shown by dot plot. Mean number is also indicated $(* * p<0.01, * * * p<0.001$; one-way ANOVA and Tukey's test)

$\beta 2$ signaling pathway might be involved in ccRCC cells (Supplementary Figure S3B). Luciferase reporter assays using a Smad binding motif $(9 \times$ CAGA-luc $)$ showed that TGF- $\beta 2$ activated Smad signaling in OS-RC- 2 cells less potently than TGF- $\beta 1$ and TGF- $\beta 3$ (Fig. $3 a$ ). Smad 2 and Smad 3 were phosphorylated by TGF- $\beta 1$ or TGF- $\beta 3$ in OSRC-2 cells, but only weakly by TGF- $\beta 2$ (Supplementary Figure S4A). However, the responsiveness to TGF- $\beta 2$ was as remarkable as that to TGF- $\beta 3$ in Caki- 1 and HK- 2 cells, which expressed higher levels of TGFBR3 (Fig. 3a). Phosphorylation of Smad2 and Smad3 was induced by TGF- $\beta 2$, as well as by TGF- $\beta 1$ or TGF- $\beta 3$ in Caki- 1 and HK-2 cells (Supplementary Figure S4A).

To directly evaluate whether downregulation of TGFBR 3 attenuates TGF- $\beta$ signaling, TGFBR 3 was either silenced or overexpressed in ccRCC cells. $9 \times$ CAGA-luc reporter assays revealed that TGF- $\beta 2$ signaling activity was significantly higher in OS-RC-2-TGFBR3 cells (Fig. 3b), whereas knockdown of TGFBR3 significantly attenuated TGF- 32 signaling in Caki-1 cells (Fig. 3c). Smad2 and Smad3 phosphorylation was also examined in OS-RC-2 cells with or without expression of TGFBR3. Although TGF- $\beta 2$ potently phosphorylated Smad 2 and Smad 3 in the presence of TGFBR3 compared to its absence, TGF- $\beta 1 / 3$ induced $\operatorname{Smad} 2 / 3$ phosphorylation was only slightly enhanced in OS-RC-2-TGFBR3 cells (Fig. 3d). In contrast, the phosphorylation of Smad $2 / 3$ by TGF- $\beta 2$ in Caki- 1 cells was reduced upon TGFBR3 silencing, while that by TGF$\beta 1$ or TGF- $\beta 3$ was not apparently altered (Supplementary Figure S4B). These results suggested that TGFBR3 mainly facilitates TGF- $\beta 2$-Smad signaling, at least under these concentrations of TGF- $\beta 2$, in ccRCC cells.

We also investigated the effect of TGF- $\beta$ signaling on tumor-forming ability. Colony-formation assays showed that TGF- $\beta 1$ and TGF- $\beta 3$ significantly decreased the colony size of OS-RC- 2 cells (Fig. 3e). In contrast to TGF- $\beta 1$ and TGF- $\beta 3$, TGF- $\beta 2$ decreased the colony size in OS-RC-2 cells only after introduction of TGFBR3 (Fig. 3e), consistent with the role of TGFBR3 in TGF- $\beta 2$ signaling. These results suggested that loss of TGFBR 3 expression mainly impairs the tumor-suppressive role of TGF- $\beta 2$, resulting in enhanced tumor-forming ability in ccRCC cells.

\section{TGF- $\beta$-TGFBR3 signaling diminishes the ALDH ${ }^{+}$ highly tumorigenic cell population in CCRCC}

To identify the genes important for tumor-forming ability among the target(s) of TGF- $\beta$, RNA-sequence (RNA-seq) analysis was performed. Candidate genes regulated by TGF- $\beta 2-T G F B R 3$ signaling were screened as shown in Fig. 4a. As a result, ALDHIAl was selected among the eight candidate genes, as it is known to be involved in stemness of certain CICs. Expression of ALDHIAl mRNA was downregulated by TGF- $\beta 2$ in OS-RC-2-GFP cells, which was enhanced in OS-RC-2-TGFBR3 cells (Fig. 4b). Although introduction of TGFBR3 decreased the basal expression of ALDH1Al mRNA, treatment with the T $\beta R I$ inhibitor, SB431542, rescued this downregulation, suggesting that the regulation of $A L D H 1 A 1$ is dependent on the TGF- $\beta$-T $\beta$ RI signaling pathway (Fig. 4b). Caki-1shTGFBR3 \#B cells, in which TGFBR3 expression was strongly silenced, were insensitive to TGF- $\beta 2$-mediated downregulation of ALDHIA1 mRNA, consistent with the findings obtained by OS-RC-2 cells (Fig. 4c). Aldefluor assays revealed that TGFBR3 overexpression in OS-RC-2 cells enhanced the TGF- $\beta 2$-mediated reduction of $\mathrm{ALDH}^{+}$ cell population, which was partially rescued by SB431542 treatment (Fig. 4d).

ALDH is a known CIC marker in many types of cancer. To determine whether ALDH1A1 serves as a marker for renal CICs, ALDH-positive OS-RC-2 (OS-RC-2 ALDH ${ }^{+}$) and ALDH-negative OS-RC-2 (OS-RC-2 $\mathrm{ALDH}^{-}$) cells were sorted. The population exhibiting high ALDH activity, which was diminished by diethylaminobenzaldehyde (DEAB), was sorted as OS-RC-2 $\mathrm{ALDH}^{+}$by Aldefluor assays (Fig. 5a). Expression of ALDHIAl and ALDH1A2 was higher in OS-RC-2 $\mathrm{ALDH}^{+}$cells than in OS-RC-2 $\mathrm{ALDH}^{-}$cells (Fig. 5b). As expression of TGFBR3 tended to be lower in $\mathrm{ALDH}^{+}$Caki-1 cells than in $\mathrm{ALDH}^{-}$cells, 
A

\section{OS-RC-2}


HK-2



TGF- $\beta 1 \quad \square$ TGF- $\beta 2 \quad \square$ TGF- 33


E



TGF- $\beta 1$



TGF- $\beta 2$



TGF- $\beta 3$



D


Fig. 3 TGFBR3 is required for the tumor-suppressive role of TGF- $\beta 2$ in ccRCC. a Responsiveness to each TGF- $\beta$ (0.03, $0.1,0.3,1,3$, and $10 \mathrm{ng} / \mathrm{ml}$ ) in indicated cells was evaluated by reporter luciferase assays. Cells were stimulated with TGF- $\beta$ s for $3 \mathrm{~h}$. Data represent individual plots of triplicate samples normalized with untreated controls. b Responsiveness to TGF- $\beta 2$ in OS-RC- 2 cells was evaluated by reporter luciferase assays. Cells were stimulated with TGF- $\beta 2$ for $3 \mathrm{~h}$. Data represent the mean number from quadruplicate samples $+\mathrm{SD}$ $(* * * p<0.001$; one-way ANOVA with Tukey's test). c Responsiveness to TGF- $\beta 2$ in Caki- 1 cells was evaluated by reporter luciferase assays. Cells were stimulated with TGF- $\beta 2$ for $3 \mathrm{~h}$. Data represent mean number from quadruplicate samples $+\mathrm{SD}(* * * p<0.001$; oneway ANOVA with Tukey's test). d Phosphorylation of Smad2 and Smad3 in OS-RC-2 cells was analyzed by immunoblotting. Cells were stimulated with TGF- $\beta$ s for $1 \mathrm{~h}$. Numbers under each blot represent fold changes by TGF- $\beta$ stimulation. e (Left) Tumor-forming ability of OS-RC-2-GFP and OS-RC-2-TGFBR3 cells was evaluated by colonyformation assays. Cells were stimulated with TGF- $\beta$ s for 17 days. (Right) Colony size is converted to base 10 logarithmic scale and shown by box and whisker plots $(* * p<0.01, * * * p<0.001$, n.s. not significant; one-way ANOVA with Sidak's test). Scale bar $=200 \mu \mathrm{m}$ 
A



B


D





BAA

Fig. 4 ALDH activity in ccRCC cells is regulated by TGF- $\beta 2-$ TGFBR3 signaling through transcriptional suppression of ALDH1A1. a Tumor-initiating cell-associated genes were screened by RNA-seq analysis. Caki-1-shNTC cells and Caki-1-shTGFBR3 \#B cells were stimulated with TGF- $\beta 2$ for $24 \mathrm{~h}$. The reads per kilobase of exon per million mapped sequence reads (RPKM) was calculated for each gene to compensate the number of transcript fragment based on the length of genes. Candidate genes were screened based on the following criteria: (i) (RPKM value in non-stimulated-Caki-1-shNTC cells) $>5$ and (RPKM value in TGF- $\beta 2$-stimulated Caki-1-shNTC cells)/(RPKM value in non-stimulated-Caki-1-shNTC cells) $<-2$; (ii) (RPKM value in TGF- 32 -stimulated-Caki-1-shTGFBR3 \#B cells) $>5$ and (RPKM value in TGF- $\beta 2$-stimulated-Caki-1-shTGFBR3 \#B cells)/(TGF- $\beta 2$ stimulated Caki-1-shNTC cells) $>2$. (Top) The red diagram shows gene clusters screened by criteria (i). The blue diagram shows gene clusters screened by criteria (ii). (Bottom) The eight overlapping candidate genes, between the two sets, are indicated. b Expression of ALDH1A1 mRNA in OS-RC-2-GFP and OS-RC-2-TGFBR3 cells was quantified by qRT-PCR analysis. Cells were stimulated with TGF- $\beta 2$



(T 32 ) or treated with SB431542 (SB) for $24 \mathrm{~h}$. Data represent the mean number normalized to SB431542-treated OS-RC-2-GFP cells from biologically quadruplicate samples $+\mathrm{SD}(* p<0.05$, $* * p<0.01$, *** $p$ $<0.001$; one-way ANOVA with Sidak's test). c Expression of ALDH1A1 mRNA in Caki-1-shNTC and Caki-1-shTGFBR3 cells was quantified by qRT-PCR analysis. Cells were stimulated with TGF- $\beta 2$ for $24 \mathrm{~h}$. Data represent the mean number normalized to nonstimulated Caki-1-shTGFBR3 \#B cells from biologically triplicate samples $+\mathrm{SD} \quad(* p<0.05, \quad * * * p<0.001 ;$ one-way ANOVA with Sidak's test). d ALDH activity in OS-RC-2-mCherry and OS-RC-2TGFBR3 cells was measured by Aldefluor assays. (Left) Cells were stimulated with TGF- $\beta 2$ or treated with SB431542 every 2 days, for up to 4 days, and subjected to Aldefluor assays. The representative percentage of $\mathrm{ALDH}^{+}$cells within the total viable cell population is indicated. (Right) Percentage of $\mathrm{ALDH}^{+}$cells in the left panel is normalized to DMSO-treated cells. Data represent the mean number from technically triplicate samples $\pm \mathrm{SD}(* * * p<0.001$, n.s. not significant; one-way ANOVA with Tukey's test) 
A





B


C
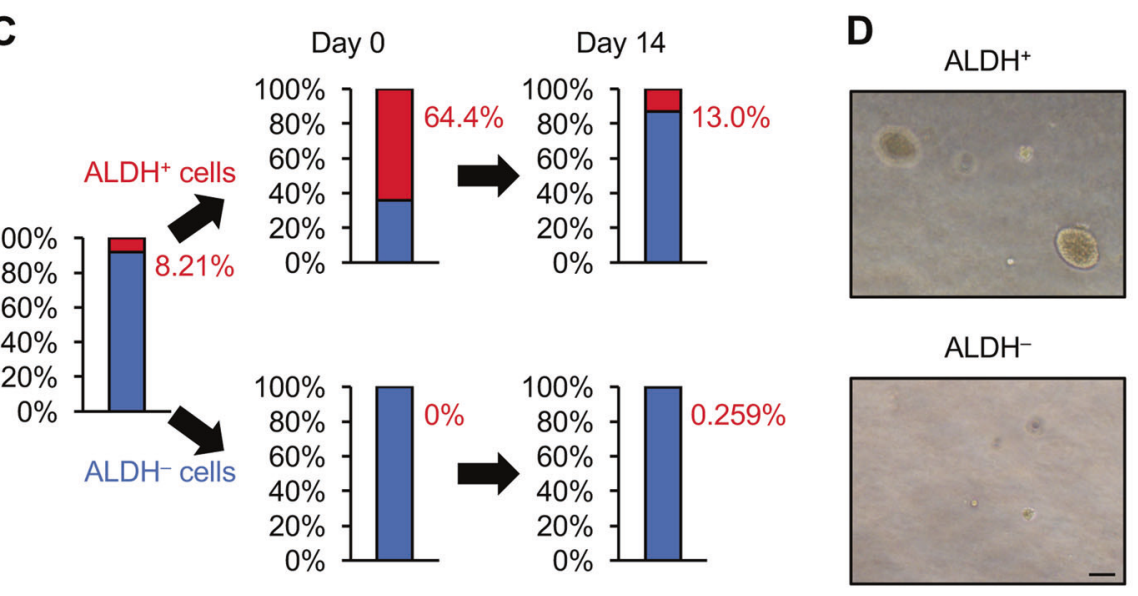

$\mathrm{ALDH}^{-}$

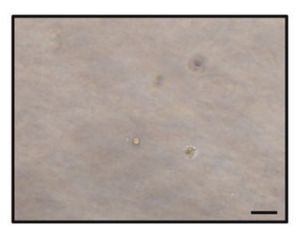

$\left[\mu \mathrm{m}^{2}\right]$



$\mathbf{E}$

OS-RC-2


$\mathrm{ALDH}^{-}$



$[\mathrm{ph} / \mathrm{s}]$



$[\mathrm{ph} / \mathrm{s}]$



heterogeneous expression of TGFBR3 might contribute to the generation of $\mathrm{ALDH}^{+}$tumor-initiating cells (Supplementary Figure S5A). According to the cancer stem-cell theory, CICs can undergo asymmetric cell division in addition to potentiating high tumor-forming ability. OS-RC$2 \mathrm{ALDH}^{+}$cells differentiated into OS-RC-2 $\mathrm{ALDH}^{-}$cells, whereas OS-RC-2 $\mathrm{ALDH}^{-}$cells did not produce OS-RC-2 
Fig. 5 CICs in ccRCC cells are enriched in the $\mathrm{ALDH}^{+}$cell population. a The fraction of OS-RC-2 $\mathrm{ALDH}^{+}$and that of OS-RC-2 $\mathrm{ALDH}^{-}$ cells were analyzed by Aldefluor assays. The percentage of OS-RC-2 $\mathrm{ALDH}^{+}$cells (red) or OS-RC-2 $\mathrm{ALDH}^{-}$cells (blue) within the total viable cell population is indicated. b Expression of $A L D H 1 A 1$ and ALDH1A2 mRNA in OS-RC-2 $\mathrm{ALDH}^{+}$and OS-RC-2 $\mathrm{ALDH}^{-}$cells was quantified by qRT-PCR analysis. Data represent the mean number from technically duplicate samples + SD. c Differentiation ability of the $\mathrm{ALDH}^{+}$cells was analyzed by Aldefluor assays. OS-RC-2 ALDH ${ }^{+}$ cells and OS-RC-2 $\mathrm{ALDH}^{-}$cells were sorted and cultured for 14 days, followed by Aldefluor assays. The percentage of OS-RC-2 ALDH ${ }^{+}$ cells (red) within the total viable cell population is indicated. $\mathbf{d}$ (Left) Tumor-forming ability of OS-RC-2 $\mathrm{ALDH}^{+}$and OS-RC-2 $\mathrm{ALDH}^{-}$ cells was evaluated by colony-formation assays. Cells were incubated for 19 days. (Right) Colony size is converted to base 10 logarithmic scale and shown by box and whisker plots $\left(*^{* *} p<0.001\right.$; Welch's $t$ test). Scale bar $=200 \mu \mathrm{m}$. e Serial transplantation of OS-RC-2 $\mathrm{ALDH}^{+}$ cells. (Top) Tumor progression of the first filial generation of OS-RC-2 $\mathrm{ALDH}^{+}$and OS-RC-2 $\mathrm{ALDH}^{-}$cells was analyzed using a mouse renal orthotopic tumor model. Primary tumors were detected by in vivo bioluminescence imaging $(n=13) 15$ days after inoculation. Overall luminescence in each mouse is converted to base 10 logarithmic scale and shown by dot plot. Basal overall luminescence is set to 1 . Mean number is also indicated $(* p<0.05$; Welch's $t$ test). (Bottom) Tumor progression of the second filial generation of OS-RC-2 ALDH ${ }^{+}$and OS-RC-2 $\mathrm{ALDH}^{-}$cells was analyzed using a mouse renal orthotopic tumor model by in vivo bioluminescence imaging $(n=10) 15$ days after inoculation. Overall luminescence in each mouse is converted to base 10 logarithmic scale and shown by dot plot. Basal overall luminescence is set to 1 . Mean number is also indicated $\left(^{*} p<0.05\right.$; Welch's $t$ test)

$\mathrm{ALDH}^{+}$cells, suggesting that OS-RC-2 $\mathrm{ALDH}^{+}$cells possess the ability to divide asymmetrically (Fig. 5c).

We next characterized the tumor-forming ability of sorted OS-RC-2 $\mathrm{ALDH}^{+}$cells in cell culture and in vivo. Colony-formation assays indicated that OS-RC-2 $\mathrm{ALDH}^{+}$ cells have significantly higher colony-forming ability than OS-RC-2 ALDH ${ }^{-}$cells (Fig. 5d). In addition, OS-RC-2 $\mathrm{ALDH}^{+}$cells formed larger tumors with a high frequency compared to OS-RC-2 $\mathrm{ALDH}^{-}$cells in an orthotopic mouse model (Fig. 5e; Supplementary Figure S5B). To confirm whether $\mathrm{ALDH}^{+}$cells in the tumor sustained cancerinitiating property, cells in the tumor tissues were re-sorted into $\mathrm{ALDH}^{+}$and $\mathrm{ALDH}^{-}$cells and subjected to serial transplantation. The second filial generation of OS-RC-2 $\mathrm{ALDH}^{+}$cells also potentiated essentially the same tumorforming properties as the first filial generation in the orthotopic mouse model (Fig. 5e; Supplementary Figure $\mathrm{S} 5 \mathrm{~B})$. These results suggested that the renal CIC population is enriched in $\mathrm{ALDH}^{+}$cells.

\section{Loss of TGFBR3 enhances the cell motility of cCRCC cells}

As TGF- $\beta$ promotes cell motility by inducing EMT, we next focused on the effect of decreased TGFBR3 expression on cell migration in ccRCC cells. Attenuation of TGF$\beta 2$ signaling was expected to decrease the migratory ability of Caki-1 cells. However, their lung metastatic ability was enhanced by TGFBR3 silencing (Fig. 2f). To investigate the underlying mechanism, we evaluated the effect of TGFBR3 downregulation on cell motility. Gene ontology analysis was performed based on RNA-seq analysis. Surprisingly, several gene clusters involved in "Cell migration", "Cell adhesion", and "Extracellular matrix organization" were enriched in Caki-1-shTGFBR3 cells (Fig. 6a). In addition, chamber migration and wound closure assays showed that cellular invasion and migration were enhanced in Caki-1shTGFBR3 cells in cell culture (Fig. 6b, c). These results suggested that loss of TGFBR3 increases the migratory ability of ccRCC cells.

We next determined whether TGF- $\beta$ signaling is involved in this observation. Chamber migration and wound closure assays revealed that cellular invasion and migration were sustained even when cells were treated with SB431542 (Fig. 6b, c). Although loss of TGFBR3 expression impaired TGF- $\beta 2$-mediated phosphorylation of Smad2/ 3 (Supplementary Figure S4B), expression of certain mesenchymal markers including fibronectin and Slug was upregulated in Caki-1-shTGFBR3 cells, even in the absence of exogenous TGF- $\beta 2$ (Fig. 6d, e). Expression of these markers was not decreased when Caki-1 cells were treated with SB431542 (Fig. 6f, g), suggesting that this mechanism is independent of the TGF- $\beta$-T $\beta$ RI signaling pathway. These results showed that loss of TGFBR3 enhanced migratory ability of ccRCC cells in a TGF- $\beta$-independent manner.

\section{Loss of TGFBR3 induces lamellipodium formation and activates FAK-PI3K-Akt signaling}

To explore the mechanism through which loss of TGFBR3 enhances cell migration, we focused on cell morphology and expression of focal adhesion marker. Immunocytochemical analysis revealed that lamellipodia with focal adhesions, indicated by the focal adhesion marker, vinculin, were formed at the edge of Caki-1 cells (Fig. 7a). Caki-1shTGFBR3 cells exhibited a round-shaped morphology with lamellipodium formation, whereas Caki-1-shNTC cells were spindle-shaped (Fig. 7a). Increased lamellipodium formation was not observed in Caki-1 cells expressing a dominant negative T $\beta$ RII (dnT $\beta$ RII) or in Caki-1-shNTC cells treated with SB431542 (Supplementary Figure S6A, B). In addition, the formation of lamellipodia in Caki-1shTGFBR3 cells was not attenuated by SB431542 treatment (Supplementary Figure S6B). These results suggested that lamellipodium formation in TGFBR3-silenced cells is also independent of TGF- $\beta$-T $\beta$ RI signaling. 
A

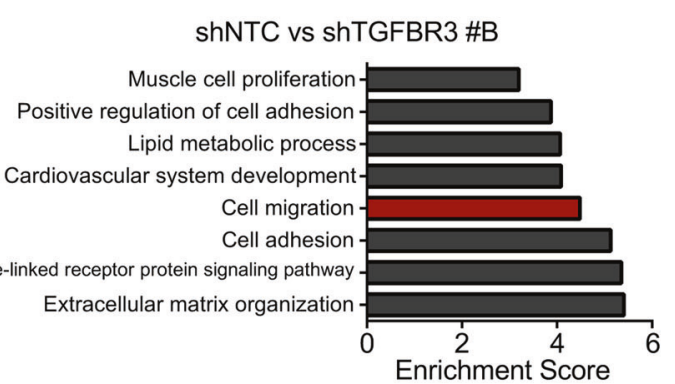

B

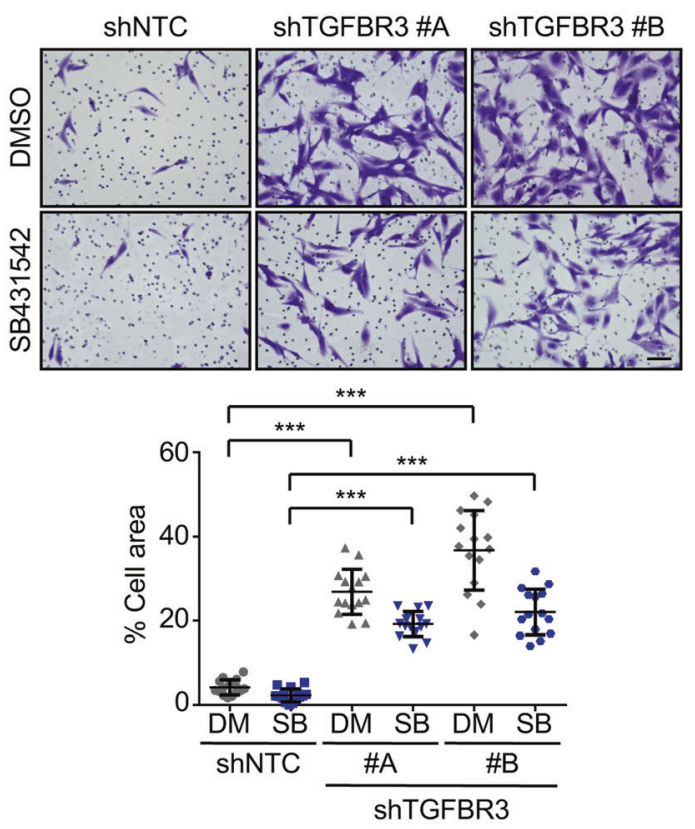

C
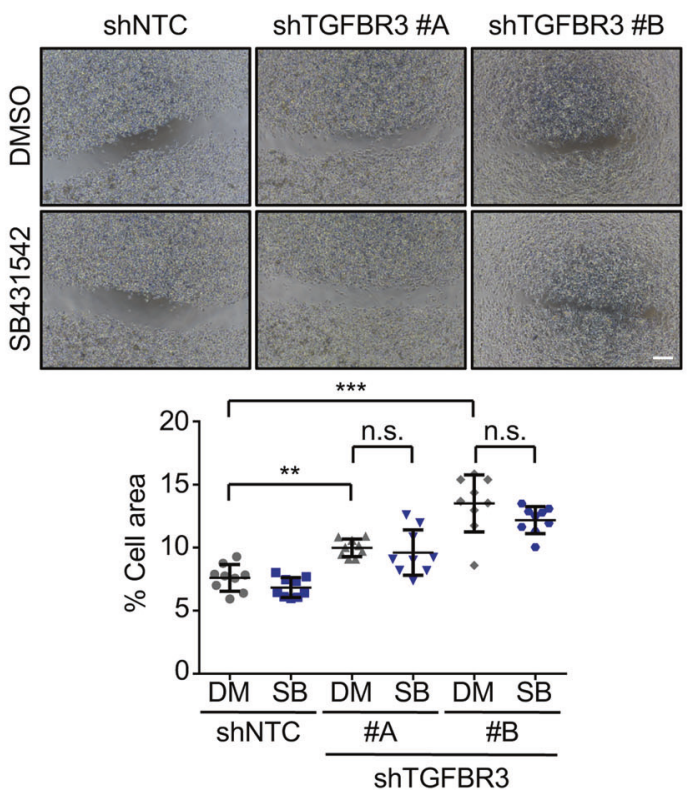

Lamellipodia are formed at the leading edge of migrating cells. In fact, time-lapse imaging showed that cells migrated in the direction of lamellipodium formation (Fig. 7b).
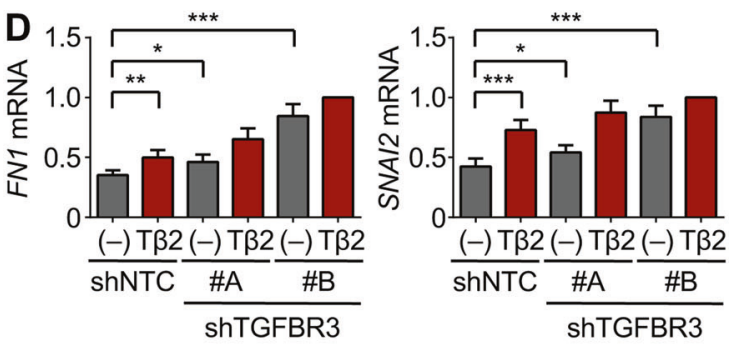

E
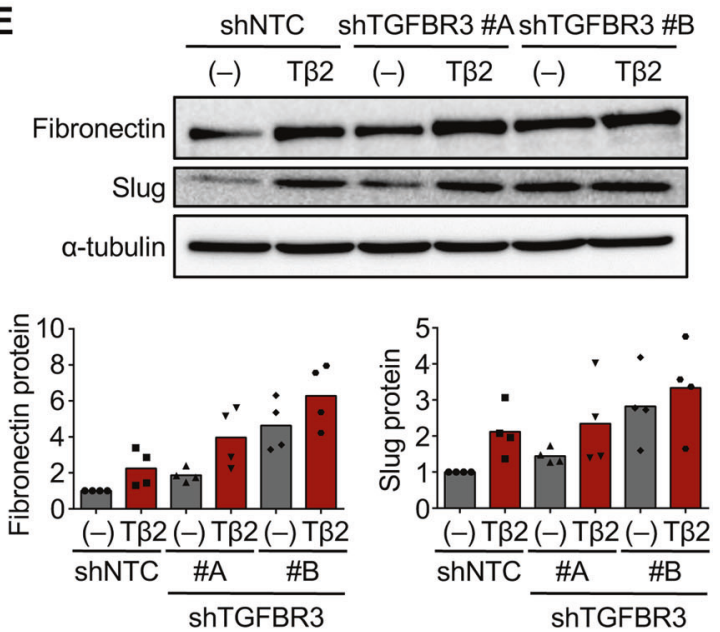

$\mathbf{F}$
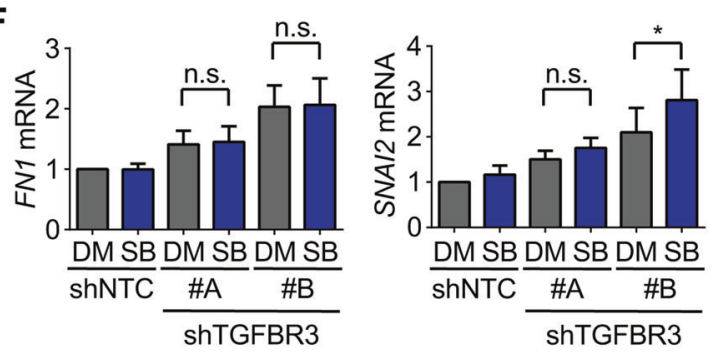

G

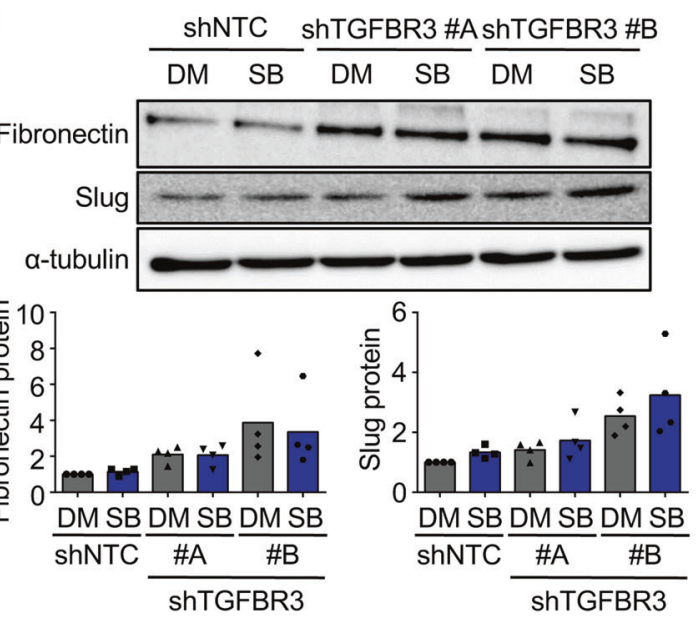

Spindle-shaped Caki-1-shNTC cells formed lamellipodia at their edges, indicative of one-dimensional migration. In contrast, Caki-1-shTGFBR3 cells formed broad 
Fig. 6 Loss of TGFBR3 enhances cell migration in ccRCC cells in a TGF- $\beta$-independent manner. a Gene functional classification was performed using DAVID. Upregulated genes in Caki-1-shTGFBR3 cells were extracted based on the following criteria: (RPKM value in Caki-1-shTGFBR3 \#B cells) $>5$ and (RPKM value in non-stimulatedCaki-1-shTGFBR3 \#B cells)/(non-stimulated-Caki-1-shNTC cells) $>1.5$. Functional annotation clustering was performed using GOTERM_BP_FAT. The enrichment score of each annotation cluster is indicated. Representative ontology for each cluster is shown. b (Top) Migratory ability of Caki-1-shNTC and Caki-1-shTGFBR3 cells was determined by chamber migration assays. Cells were treated with SB431542 for $16 \mathrm{~h}$. (Bottom) Percent area covered by crystal violetpositive cells was measured using FIJI and is shown by dot plots (*** $p<0.001$; one-way ANOVA with Tukey's test). Scale bar $=100$ $\mu \mathrm{m}$. c (Top) Invasive ability of Caki-1-shNTC and Caki-1-shTGFBR3 cells was determined by wound closure assays. Cells were treated with SB431542 for $8 \mathrm{~h}$ after cell monolayers were wounded. (Bottom) The percentage of wound closure (area) was measured using FIJI and is shown by dot plots $(* * p<0.01, * * * p<0.001, n$.s. not significant; one-way ANOVA with Tukey's test). Scale bar $=200 \mu \mathrm{m}$. d Expression of FN1 (gene encoding fibronectin) and SNAI2 (gene encoding Slug) mRNA in Caki-1-shNTC and Caki-1-shTGFBR3 cells was quantified by qRT-PCR analysis. Cells were stimulated with TGF- $\beta 2$ for $24 \mathrm{~h}$. Data represent the mean number normalized to TGF- $\beta 2-$ stimulated Caki-1-shTGFBR3 \#B cells from biologically sextuplicate samples + SD $\quad(* p<0.05, \quad * * p<0.01, \quad * * * p<0.001 ; \quad$ one-way ANOVA with Sidak's test). e (Top) Expression of fibronectin, Slug, and $\alpha$-tubulin in Caki-1-shNTC and Caki-1-shTGFBR3 cells was detected by immunoblotting. Cells were stimulated with TGF- $\beta 2$ for 24 h. T $\beta 2$ : TGF- $\beta 2$. (Bottom) Each blot was quantified by FIJI. Data represent the mean number normalized to control cells from biologically quadruplicate samples. f Expression of $F N 1$ and SNAI2 mRNA in Caki-1-shNTC and Caki-1-shTGFBR3 cells was quantified by qRTPCR analysis. Cells were treated with SB431542 for $24 \mathrm{~h}$. Data represent the mean number normalized to SB431542-treated Caki-1shTGFBR3 \#B cells from biologically quintuplicate samples + SD $\left({ }^{*} p<0.05, n . s\right.$. not significant; one-way ANOVA with Sidak's test). $\mathbf{g}$ (Top) Expression of fibronectin, Slug, and $\alpha$-tubulin in Caki-1-shNTC and Caki-1-shTGFBR3 cells was detected by immunoblotting. Cells were treated with SB431542 for $24 \mathrm{~h}$. DM: DMSO, SB: SB431542. (Bottom) Each blot was quantified by FIJI. Data represent the mean number normalized to control cells from biologically quadruplicate samples

lamellipodia and migrated two-dimensionally (Fig. 7b). These results suggested that loss of TGFBR3 induces lamellipodium formation, which in turn endows RCC cells with multi-directional cell-migratory ability.

Finally, we determined whether focal adhesion kinase (FAK) signaling was involved in lamellipodium formation. FAK phosphorylation was upregulated in Caki-1shTGFBR 3 cells, compared to that in Caki-1-shNTC cells (Fig. 7c), and this was not altered by TGF- $\beta 2$ or SB431542 (Supplementary Figure S6C). PI3K-Akt signaling is a downstream target of FAK. Akt was also phosphorylated in Caki-1-shTGFBR3 cells, showing that FAK signaling was upregulated. In addition, treating Caki-1-shTGFBR3 cells with the FAK inhibitor PF562271 attenuated Akt phosphorylation (Fig. 7c). To validate the role of PI3K signaling in lamellipodium formation, PI3K signaling was inhibited in Caki-1 cells. Treatment of Caki-1-shTGFBR3 cells with the PI3K inhibitor LY294002 led to lamellipodium regression (Fig. 7d, e; Supplementary Figure S6D). Caki-1shTGFBR3 cells were also introduced with dominant negative mutant of PI3K component p85 (dp85), which lacks the binding site for the catalytic subunit p110 [18]. Introduction of dp85 inhibited phosphorylation of Akt and significantly decreased the roundness of Caki-1-shTGFBR3 cells, consistent with the results obtained by the treatment with LY294002 (Supplementary Figure S6E, F). These results suggested that FAK-PI3K signaling has an important role in enhanced lamellipodium formation with TGFBR3 downregulation.

\section{Discussion}

A previous study reported low expression of TGFBR3 in ccRCC patients based on small number of patient samples [16]. In the present study, we found that expression of TGFBR3 was reduced in ccRCC in a clinical stagedependent manner by analyzing large number of patient samples from TCGA data set. Moreover, we demonstrated for the first time that silencing of TGFBR3 enhances primary tumor formation and metastasis of ccRCC cells using in vivo mouse model. Thus, our findings clearly imply the clinical significance of decreased expression of TGFBR3 in ccRCC. Further experiments in cell culture indicated that loss of TGFBR3 contributes to a metastatic phenotype in ccRCC cells by modulating tumor-forming and cellmigratory abilities through distinct pathways (Fig. 8). To our knowledge, there were no reports describing the role of TGFBR3 on the maintenance of CICs. In addition, although downregulation of TGFBR 3 attenuated TGF- $\beta 2$ signaling, expression of some mesenchymal markers was upregulated rather than downregulated. These findings uncovered novel compensatory mechanisms for acquiring a metastatic phenotype even after cancer cells become resistant to the growth inhibitory effect of TGF- $\beta$.

In ccRCC, several molecules are proposed to be markers of CICs [19]. Previous reports showed that endoglin (encoded by $E N G$ gene), also known as CD105, is a CIC marker of ccRCC [20]. Endoglin is a TGF- $\beta$ type III receptor, similar to TGFBR3, and functions as an accessory protein for bone morphogenetic protein-9 (BMP-9)-ALK1 signaling [21, 22]. Analyzing a TCGA data set, we have found that TGFBR3 was downregulated in ccRCC tissues, whereas $E N G$ was upregulated, suggesting that TGFBR3 and endoglin have distinct roles in the progression of ccRCC. Endoglin also has a role in modulating TGF- $\beta$ Smad1/5/8 signaling, resulting in attenuated TGF- $\beta$-Smad $2 /$ 3 signaling [23]. Thus, downregulation of TGFBR3 and upregulation of endoglin may cause attenuation of TGF- $\beta$ Smad2/3 signaling and enhancement of tumor-forming 
A shNTC
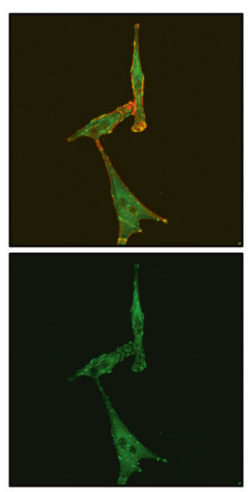

ShTGFBR3 \#A
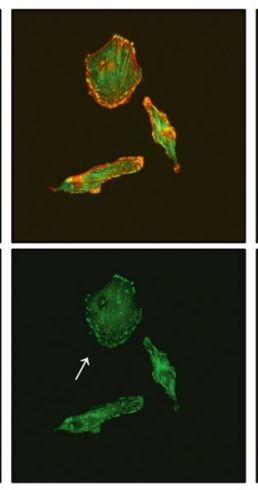

Phalloidin / vinculin
shTGFBR3 \#B
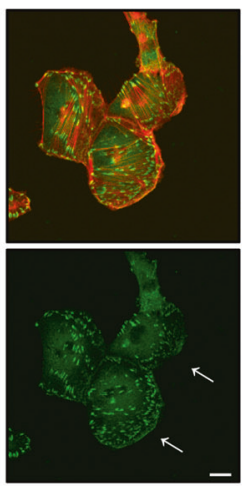

C

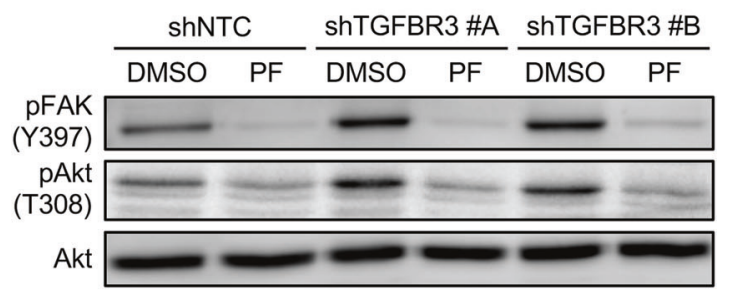

B
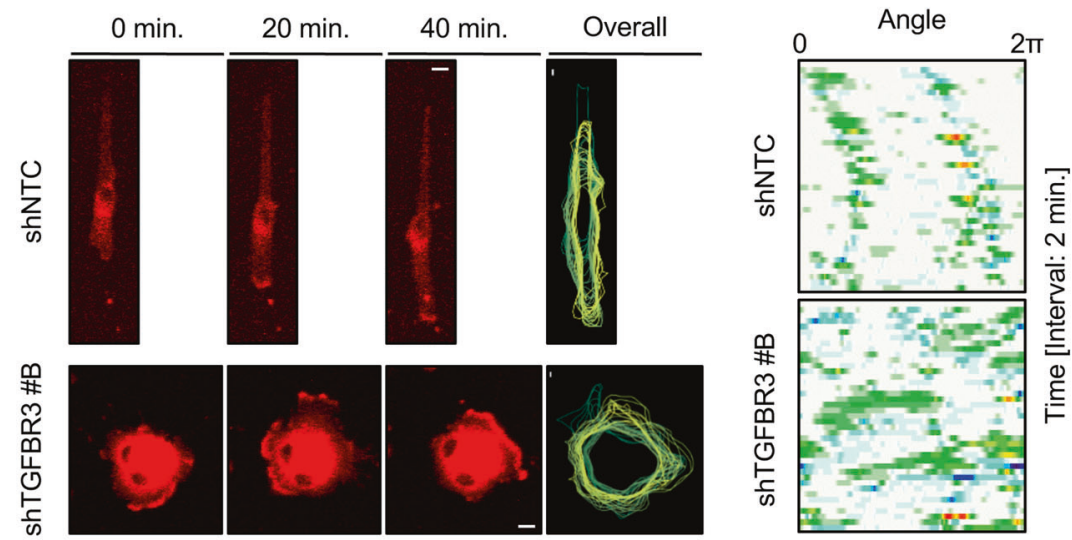

[Extension]

$8.9 \times 10^{-6}$

Actin-RFP

D

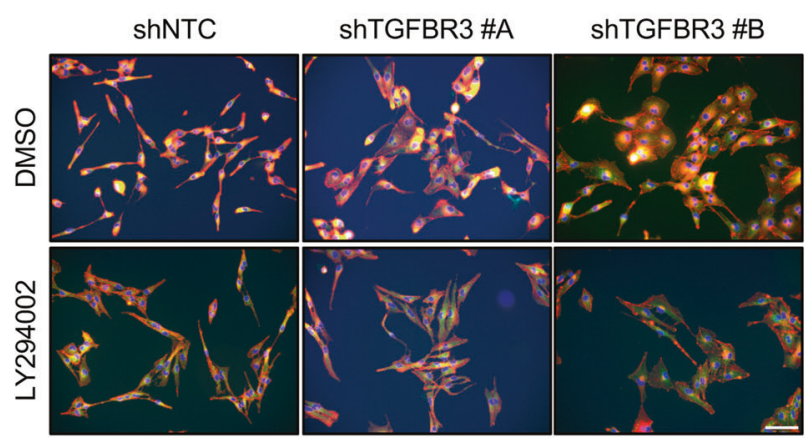

DAPI / Phalloidin / vinculin

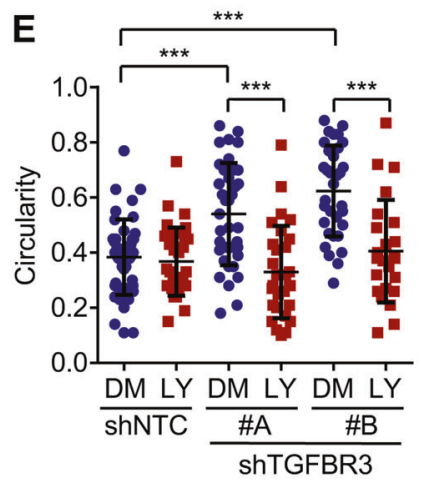

Fig. 7 Loss of TGFBR3 increases lamellipodium formation in ccRCC cells through FAK-PI3K signaling. a The morphology of Caki-1shNTC and Caki-1-shTGFBR3 cells was observed by immunocytochemistry. Arrows show the accumulation of focal adhesions at the edge of cells (lamellipodia). Scale bar $=20 \mu \mathrm{m}$. Red: phalloidin, green: vinculin. b Cell migration was observed by time-lapse imaging. (Left) Morphology of Caki-1-shNTC and Caki-1-shTGFBR3 \#B cells, indicated every $20 \mathrm{~min}$. Scale $b a r=1 \mu \mathrm{m}$. Sequential single-cell morphology was observed every $2 \mathrm{~min}$. Red represents Actin-RFP expression using the baculovirus system. (Right) Kymogragh of cell migration used to visualize the morphological changes at the invasive front. Morphological changes were observed every $2 \mathrm{~min}$, for up to 80

min, which are indicated as "time" on the vertical axis. The horizontal axis shows each segmented cell outline of Caki-1-shNTC and Caki-1shTGFBR3 \#B cells. Warm and cold colors represent extension and retraction of the invasive front, respectively. c Phosphorylated FAK (Y397) and phosphorylated Akt (T308) in Caki-1-shNTC and Caki-1shTGFBR 3 cells were analyzed by immunoblotting. Cells were treated with PF562271 for 36h. PF: PF562271. d Morphology of Caki-1shNTC and Caki-1-shTGFBR3 cells was observed by immunocytochemistry. Cells were treated with LY294002 for $36 \mathrm{~h}$. Scale bar = $100 \mu \mathrm{m}$. e Cell morphology was quantified based on circularity. Images were processed using FIJI $(* * * p<0.001$; one-way ANOVA with Tukey's test). DM: DMSO, LY: LY294002 


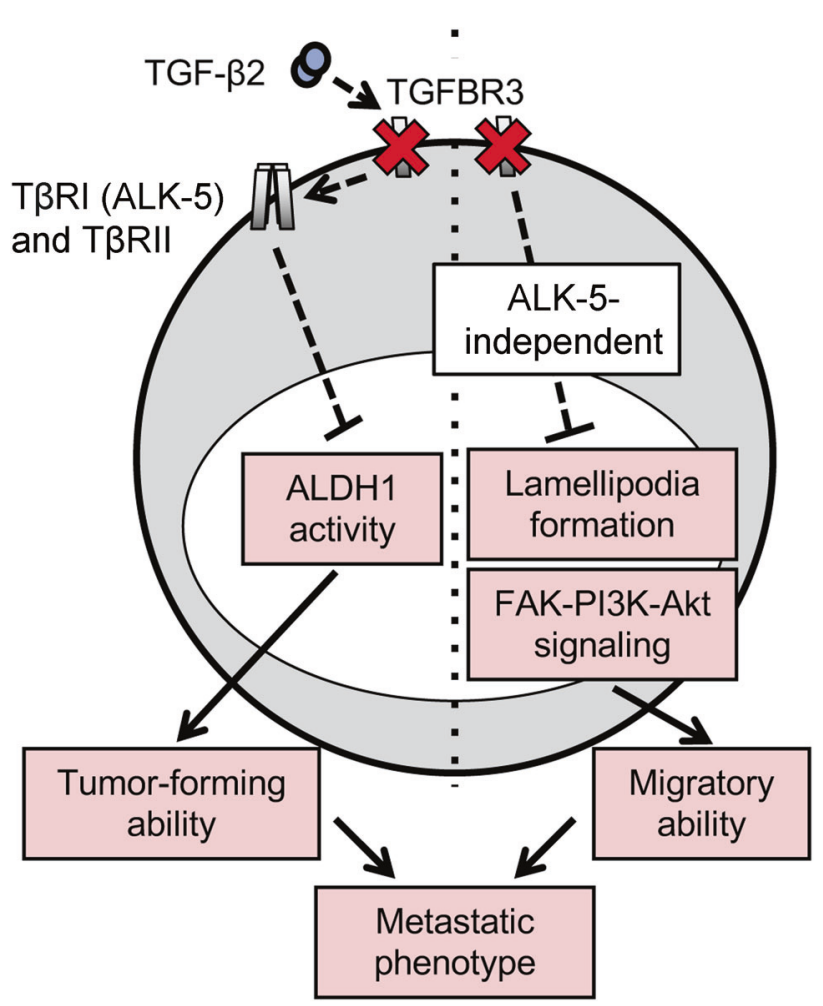

Fig. 8 Decreased expression of TGFBR3 induces tumor-forming and migratory ability in ccRCC cells. Loss of TGFBR3 enhances the metastatic phenotype of ccRCC cells through distinct pathways. Tumor-forming ability is regulated by the TGF- $\beta 2$-TGFBR3-ALDH pathway in the CIC population. Cell migration is regulated by TGFBR3 through lamellipodium formation and the FAK-PI3K signaling pathway in a TGF- $\beta$-T $\beta$ RI-independent manner

ability in ccRCC cells, suggesting that TGF- $\beta$-Smad $2 /$ 3 signaling is important to inhibit the tumor-forming ability of ccRCC cells. ALDH1 has also been reported to be a CIC marker in other types of cancer and ccRCC cell lines [24, 25]. Our findings demonstrated that CICs are enriched in the $\mathrm{ALDH}^{+}$ccRCC cell population; this effect was attenuated by TGF- $\beta 2$ unless TGFBR 3 expression was downregulated. The regulatory mechanisms of TGF- $\beta$ in maintaining the CIC population are well described in other types of cancer cells such as diffuse-type gastric cancer [26, 27] and pancreatic cancer [28]. Especially in some types of pancreatic cancer cells, ALDHIAl was reported to be a direct transcriptional target of $\operatorname{Smad} 2 / 3$ and Smad4 [28], indicating that TGF- $\beta$-Smad $2 / 3$ signaling negatively regulates the maintenance of CICs in many kinds of cancer.

Although TGFBR3 has been reported to act as both a tumor promoter and suppressor [29,30], its role appears to be closely related to the molecular form of TGFBR3 (i.e., transmembrane or soluble form). As the transmembrane form of TGFBR 3 is an accessory protein in the TGF- $\beta$ receptor complex and enhances TGF- $\beta$ signal transduction, the soluble form acts as a decoy for TGF- $\beta$ ligands, inhibiting TGF- $\beta$ signaling [31]. In this study, TGFBR3 protein in ccRCC cells was detected in the membrane fraction of cell lysates (Fig. 2a, b). Recent studies suggest that the transmembrane form of TGFBR3 has functions other than supporting the interaction between TGF- $\beta$ and T $\beta$ RII. For example, TGFBR3 binds to $\beta$-arrestin 2 and GAIP-interacting protein, $\mathrm{C}$ terminus (GIPC) through its intracellular domain to regulate endocytosis of the TGF- $\beta$ receptor complex and TGF- $\beta$ signaling [32, 33]. In ovarian cancer, loss of TGFBR3 regulates cell division cycle 42 (CDC42) activity through the regulation of $\beta$-arrestin 2 and enhances cell migration in a TGF- $\beta$ signaling-independent manner [34]. As our study revealed distinct functions for TGFBR3 in cytoskeleton dynamics, the molecular mechanisms might be explained in part by the ligandindependent role of TGFBR3.

TGFBR3 is known to bind to other members of the TGF$\beta$ family. Inhibin, a heterodimer of inhibin $\alpha$ - and $\beta$-chains, binds to TGFBR3 and inhibits the activity of activin, a homodimer of inhibin $\beta$-chains [35]. SB431542 inhibits the kinase activity of T $\beta R I$ as well as that of type I receptors of activin (ALK-4 and ALK-7). As SB431542 failed to inhibit the production of fibronectin and Slug (Fig. 6f, g), lamellipodium formation, or FAK activation (Supplementary Figure S6B, C), neither inhibin nor activin are involved in these processes in ccRCC cells. TGFBR3 has also been reported to bind BMPs and regulate their signaling activities [36]. However, LDN193189, which blocks BMP signaling by inhibiting type I receptors, ALK-2 and ALK-3, affects neither lamellipodium formation nor FAK activation of ccRCC cells (data not shown). Therefore, BMP signaling does not appear to be involved in these processes.

With recent large-scale analyses, the central role of PI3K signaling in ccRCC progression has emerged. Integrative pathway analysis, performed by the TCGA research network, showed that PI3K-Akt signaling and the downstream mTOR pathway are aberrantly activated in ccRCC cells [37]. Other genomic analyses showed that some genes involved in PI3K-Akt-mTOR signaling and the upstream focal adhesion pathway are also mutated in nearly $60 \%$ of the ccRCC patients [38, 39]. As PI3K-Akt-mTOR signaling is known to regulate HIF signaling and facilitate angiogenesis, mTOR inhibitors, such as everolimus and temsirolimus, have been applied for the treatment of ccRCC, in addition to tyrosine kinase inhibitors [12]. However, little is known about crosstalk between PI3K signaling and other pathways during ccRCC progression. Our present study revealed that loss of TGFBR3, a component of the TGF- $\beta$ signaling pathway, affects FAK-PI3K-mediated cell migration. Further studies will unveil the contribution of TGF- $\beta$ signaling to ccRCC metastasis through the interaction with pivotal signaling pathways in ccRCC progression. 


\section{Materials and methods}

\section{Cell culture and reagents}

Human normal proximal tubule HK-2 cells (American Type Culture Collection (ATCC), Manassas, VA) were maintained in Dulbecco's modified Eagle's medium:Nutrient Mixture F-12 (DMEM/F12) medium (Thermo Fisher Scientific, Waltham, MA) containing $10 \%$ fetal bovine serum. Human embryonic kidney HEK 293 cells (ATCC) were maintained in DMEM (Thermo Fisher Scientific) containing $10 \%$ fetal bovine serum. Human clear-cell renal carcinoma cells, OS-RC-2 (RIKEN Cell Bank, Tsukuba, Japan), Caki1 (Cell Resource Center for Biomedical Research, Tohoku University, Miyagi, Japan), ACHN (ATCC), and 786-O (ATCC) were cultured as recommended by ATCC. TGF$\beta 1$, TGF- $\beta 2$, and TGF- $\beta 3$ ligands (R\&D Systems, Minneapolis, $\mathrm{MN}$ ) were reconstituted in $4 \mathrm{mM} \mathrm{HCl}$ containing $0.1 \%$ bovine serum albumin (Sigma-Aldrich, St Louis, MO) and used at a concentration of $0.3 \mathrm{ng} / \mathrm{ml}$ with Caki- 1 cells or $1 \mathrm{ng} / \mathrm{ml}$ with OS-RC-2 cells, unless otherwise mentioned. SB431542 (Sigma-Aldrich) was reconstituted in dimethyl sulfoxide and used at a concentration of $5 \mu \mathrm{M}$. LY294002 (Sigma-Aldrich) and PF562271 (Selleck Chemicals, Houston, TX) were reconstituted in dimethyl sulfoxide and used at a concentration of $20 \mu \mathrm{M}$.

\section{qRT-PCR analysis}

Total RNA was extracted using Isogen Reagent (Nippon Gene, Toyama, Japan). Reverse transcription and qRT-PCR analysis were performed as described previously [40, 41]. All samples were run in duplicate. mRNA expression was normalized to $G A P D H$ expression. Primer sequences are shown in Supplementary Table S1 and S2. Data shown are representative of at least two experiments with similar results, unless otherwise mentioned.

\section{Immunoblotting}

Total cell lysates were prepared following published methods [42]. Membrane fraction of lysates were prepared with MEM-PER Plus Membrane Protein Extraction Kit (Thermo Fisher Scientific). Immunoblotting was performed as described previously [43]. Membranes were blocked with 5\% skim milk in Tris-buffered saline with Tween (TBST) or Blocking-One reagent (Nacalai Tesque, Kyoto, Japan). Primary antibodies are listed in Supplementary Table S3. Primary antibodies were diluted with TBST or Can Get Signal 1 (Toyobo, Osaka, Japan). Data shown are representative of at least two experiments with similar results, unless otherwise mentioned.

\section{Reporter luciferase assays}

Cells were plated in 24-well plates (Thermo Fisher Scientific). After 1 day, cells were transiently transfected with plasmids using Lipofectamine 3000 (Thermo Fisher Scientific). After an additional 1-day incubation, cells were stimulated with the indicated concentrations of TGF- $\beta$ ligands for $3 \mathrm{~h}$. TGF- $\beta$ signaling was evaluated using the $9 \times$ CAGA-Luc plasmid. $9 \times$ CAGA-Luc activity was normalized to CMV-RLuc activity, as described previously [44]. Data shown are representative of at least two experiments with similar results.

\section{Aldefluor assays}

Cells were plated in $100 \times 20$-mm tissue culture dishes (Corning, Corning, NY). Aldefluor assays were performed using an ALDEFLUOR Kit (StemCell Technologies, Vancouver, Canada) as described previously [28]. $\mathrm{ALDH}^{+}$cells were analyzed and sorted with an Epics-XL, Gallios, and MoFlo Astrios (Beckman Coulter, Pasadena, CA). Data shown are representative of at least two experiments with similar results.

\section{Immunocytochemistry}

Cells were plated on Matsunami Micro Cover Glass (Matsunami, Osaka, Japan), fixed with $4 \%$ paraformaldehyde in phosphate-buffered saline (PBS), and permeabilized with $0.2 \%$ Triton-X in PBS. Cells were then stained with tetramethylrhodamine-conjugated phalloidin (SigmaAldrich), primary antibodies (Supplementary Table S3), and secondary antibodies in Blocking-One reagent (Nacalai Tesque). After TO-PRO-3 or DAPI staining for nuclear detection, cells were mounted with Dako Fluorescent Mounting Medium (DAKO, Glostrup, Denmark). Images were captured with an Axiovert 200M microscope (Zeiss, Oberkochen, Germany) or a BZ-X710 microscope (Keyence, Osaka, Japan). Image analysis was performed using FIJI software. Data shown are representative of at least two experiments with similar results.

\section{Mouse renal orthotopic tumor models}

All protocols were approved by the Animal Ethics Committee of The University of Tokyo. BALB/c-nu/nu male mice (5-weeks-old) were purchased from Sankyo Labo Service Corporation (Tokyo, Japan). Mice were anesthetized with avertin solution, which contained 2-, 2-, 2tribromoethanol (Sigma-Aldrich) and tert-Amyl alcohol (Sigma-Aldrich) in Hank's balanced salt solution (HBSS, Thermo Fisher Scientific). Renal subcapsule inoculation was described previously [45, 46]. Kidneys were exposed 
on the back of anesthetized mice. Cells were reconstituted in $50 \mu \mathrm{l}$ of HBSS. A syringe with a 29 -gauge needle (Terumo, Tokyo, Japan) was inserted from the lower pole to the subrenal capsule. After inoculation, the incision was closed with a surgical clip.

\section{Bioluminescence imaging analyses}

For in vivo bioluminescence imaging, mice were anesthetized with avertin solution and intraperitoneally injected with $2.5 \mathrm{mg}$ of D-Luciferin potassium salt (Promega, Madison, WI) dissolved in $200 \mu \mathrm{l}$ of PBS. After $10 \mathrm{~min}$, in vivo bioluminescence imaging was performed using the NightOWL LB981 (Berthold Technologies, Bad Wildbad, Germany). For ex vivo bioluminescence imaging, resected lungs were incubated with D-Luciferin solution for $5 \mathrm{~min}$ and imaged using NightOWL LB981. Images were captured and analyzed using IndiGO software (Berthold Technologies). Mice were randomly divided into groups without blinding for outcome assessment and sample size estimation. Number of mice used was indicated in each figure.

\section{RNA-Seq analysis}

RNA-seq analysis was performed as described previously [47]. A complementary DNA library was prepared using the RNeasy Mini Kit with the On-Column DNase Digestion Set (Qiagen, Venlo, Netherlands), Dynabeads mRNA DIRECT Micro Purification kit and Ion Total RNA-Seq Kit v2 (Thermo Fisher Scientific). Samples were then amplified with Ion PI Hi-Q Chef Kit and applied to an Ion PI Chip v3 in Ion Chef (Thermo Fisher Scientific). DAVID [48, 49] was used for gene functional classification. Raw and processed data are available at GEO (https://www.ncbi.nlm. nih.gov/geo/query/acc.cgi?acc $=$ GSE106719).

Acknowledgements We thank Dr. Yoko Katsuno, Dr. Anna Mizutani, Dr. Yukari Hoshino, Dr. Fumihiko Murai, Dr. Kei Takahashi, and Dr. Luna Taguchi (The University of Tokyo) for technical help; and Dr. Hiroyuki Miyoshi (Keio University) for providing the lentiviral vectors. This work was supported by KAKENHI Grants-in-Aid for Scientific Research (S) (15H05774) (KM) and Scientific Research (C) (15K08393) (SE), and Grant-in-Aid for JSPS Research Fellow (16J05993) (JN) from the Japan Society for the Promotion of Science (JSPS), and the Yasuda Medical Foundation (KM).

Author contributions JN, KM, and SE designed the study, analyzed the data, and wrote the manuscript. JN performed experiments.

\section{Compliance with ethical standards}

Conflict of interest The authors declare that they have no competing interests.
Open Access This article is licensed under a Creative Commons Attribution-NonCommercial-NoDerivatives 4.0 International License, which permits any non-commercial use, sharing, distribution and reproduction in any medium or format, as long as you give appropriate credit to the original author(s) and the source, and provide a link to the Creative Commons license. You do not have permission under this license to share adapted material derived from this article or parts of it. The images or other third party material in this article are included in the article's Creative Commons license, unless indicated otherwise in a credit line to the material. If material is not included in the article's Creative Commons license and your intended use is not permitted by statutory regulation or exceeds the permitted use, you will need to obtain permission directly from the copyright holder. To view a copy of this license, visit http://creativecommons.org/licenses/by-nc-nd/4.0/.

\section{References}

1. Massagué J, Obenauf AC. Metastatic colonization by circulating tumour cells. Nature. 2016;529:298-306.

2. Kreso A, Dick JE. Evolution of the cancer stem-cell model. Cell Stem Cell. 2014;14:275-91.

3. Morikawa M, Derynck R, Miyazono K. TGF- $\beta$ and the TGF- $\beta$ family: context-dependent roles in cell and tissue physiology. Cold Spring Harb Perspect Biol. 2016;8:a021873.

4. Heldin $\mathrm{CH}$, Miyazono K, ten Dijke P. TGF- $\beta$ signalling from cell membrane to nucleus through SMAD proteins. Nature. 1997;390:465-71.

5. Shi Y, Massagué J. Mechanisms of TGF- $\beta$ signaling from cell membrane to the nucleus. Cell. 2003;113:685-700.

6. Feng X-H, Derynck R. Specificity and versatility in TGF- $\beta$ signaling through Smads. Annu Rev Cell Dev Biol. 2005;21:659-93.

7. Bierie B, Moses HL. TGF- $\beta$ and cancer. Cytokine Growth Factor Rev. 2006;17:29-40.

8. Miyazono K, Ehata S, Koinuma D. Tumor-promoting functions of transforming growth factor- $\beta$ in progression of cancer. Ups J Med Sci. 2012;117:143-52.

9. Levy L, Hill CS. Alterations in components of the TGF- $\beta$ superfamily signaling pathways in human cancer. Cytokine Growth Factor Rev. 2006;17:41-58.

10. Katsuno Y, Lamouille S, Derynck R. TGF- $\beta$ signaling and epithelial-mesenchymal transition in cancer progression. Curr Opin Oncol. 2013;25:76-84.

11. Bardeesy N, Cheng KH, Berger JH, Chu GC, Pahler J, Olson P, et al. Smad4 is dispensable for normal pancreas development yet critical in progression and tumor biology of pancreas cancer. Genes Dev. 2006;20:3130-46.

12. Capitanio U, Montorsi F. Renal cancer. Lancet. 2016;387:894-906.

13. Gossage L, Eisen T, Maher ER, VHL, the story of a tumour suppressor gene. Nat Rev Cancer. 2015;15:55-64.

14. Vanharanta S, Shu W, Brenet F, Hakimi AA, Heguy A, Viale A, et al. Epigenetic expansion of VHL-HIF signal output drives multiorgan metastasis in renal cancer. Nat Med. 2013;19:50-56.

15. Ananth S, Knebelmann B, Gruning W, Dhanabal M, Walz G, Stillman IE, et al. Transforming growth factor $\beta 1$ is a target for the von Hippel-Lindau tumor suppressor and a critical growth factor for clear cell renal carcinoma. Cancer Res. 1999;59:2210-6.

16. Copland JA, Luxon BA, Ajani L, Maity T, Campagnaro E, Guo $\mathrm{H}$, et al. Genomic profiling identifies alterations in TGF $\beta$ signaling through loss of TGF $\beta$ receptor expression in human renal cell carcinogenesis and progression. Oncogene. 2003;22:8053-62.

17. López-Casillas F, Wrana JL, Massagué J. Betaglycan presents ligand to the TGF $\beta$ signaling receptor. Cell. 1993;73:1435-44. 
18. Dhand R, Hara K, Hiles I, Bax B, Gout I, Panayotou G, et al. PI 3kinase: structural and functional analysis of intersubunit interactions. EMBO J. 1994;13:511-21.

19. Peired AJ, Sisti A, Romagnani P. Renal cancer stem cells: characterization and targeted therapies. Stem Cells Int. 2016;2016:8342625.

20. Bussolati B, Bruno S, Grange C, Ferrando U, Camussi G. Identification of a tumor-initiating stem cell population in human renal carcinomas. FASEB J. 2008;22:3696-705.

21. Barbara NP, Wrana JL, Letarte M. Endoglin is an accessory protein that interacts with the signaling receptor complex of multiple members of the transforming growth factor- $\beta$ superfamily. J Biol Chem. 1999;274:584-94.

22. Scharpfenecker M, van Dinther M, Liu Z, van Bezooijen RL, Zhao Q, Pukac L, et al. BMP-9 signals via ALK1 and inhibits bFGF-induced endothelial cell proliferation and VEGF-stimulated angiogenesis. J Cell Sci. 2007;120:964-72.

23. Dallas NA, Samuel S, Xia L, Fan F, Gray MJ, Lim SJ, et al. Endoglin (CD105): a marker of tumor vasculature and potential target for therapy. Clin Cancer Res. 2008;14:1931-7.

24. Marcato P, Dean CA, Giacomantonio CA, Lee PWK. Aldehyde dehydrogenase: its role as a cancer stem cell marker comes down to the specific isoform. Cell Cycle. 2011;10:1378-84.

25. Ueda K, Ogasawara S, Akiba J, Nakayama M, Todoroki K, Ueda $\mathrm{K}$, et al. Aldehyde dehydrogenase 1 identifies cells with cancer stem cell-like properties in a human renal cell carcinoma cell line. PLoS ONE. 2013;8:e75463.

26. Ehata S, Johansson E, Katayama R, Koike S, Watanabe A, Hoshino $\mathrm{Y}$, et al. Transforming growth factor- $\beta$ decreases the cancerinitiating cell population within diffuse-type gastric carcinoma cells. Oncogene. 2010;30:1693-705.

27. Katsuno Y, Ehata S, Yashiro M, Yanagihara K, Hirakawa K, Miyazono K. Coordinated expression of REG4 and aldehyde dehydrogenase 1 regulating tumourigenic capacity of diffuse-type gastric carcinoma-initiating cells is inhibited by TGF- $\beta$. J Pathol. 2012;228:391-404.

28. Hoshino Y, Nishida J, Katsuno Y, Koinuma D, Aoki T, Kokudo $\mathrm{N}$, et al. Smad4 decreases the population of pancreatic cancerinitiating cells through transcriptional repression of ALDH1A1. Am J Pathol. 2015;185:1457-70.

29. Bilandzic M, Stenvers KL. Betaglycan: a multifunctional accessory. Mol Cell Endocrinol. 2011;339:180-9.

30. Bernabeu C, Lopez-Novoa JM, Quintanilla M. The emerging role of TGF- $\beta$ superfamily coreceptors in cancer. Biochim Biophys Acta. 2009; 1792:954-73.

31. Heldin $\mathrm{CH}$, Moustakas A. Signaling receptors for TGF- $\beta$ family members. Cold Spring Harb Perspect Biol. 2016;8:a022053.

32. Chen W, Kirkbride KC, How T, Nelson CD, Mo J, Frederick JP, et al. $\beta$-arrestin 2 mediates endocytosis of type III TGF- $\beta$ receptor and downregulation of its signaling. Science. 2003;301:1394-7.

33. Blobe GC, Liu X, Fang SJ, How T, Lodish HF. A novel mechanism for regulating transforming growth factor $\beta$ (TGF- $\beta$ ) signaling. Functional modulation of type III TGF- $\beta$ receptor expression through interaction with the PDZ domain protein, GIPC. J Biol Chem. 2001;276:39608-17.

34. Mythreye K, Blobe GC. The type III TGF- $\beta$ receptor regulates epithelial and cancer cell migration through $\beta$-arrestin2-mediated activation of Cdc42. Proc Natl Acad Sci USA. 2009;106:8221-6.

35. Namwanje M, Brown CW. Activins and inhibins: Roles in development, physiology, and disease. Cold Spring Harb Perspect Biol. 2016;8:a021881.

36. Kirkbride KC, Townsend TA, Bruinsma MW, Barnett JV, Blobe GC. Bone morphogenetic proteins signal through the transforming growth factor- $\beta$ type III receptor. J Biol Chem. 2008;283:7628-37.

37. Cancer Genome Atlas Research Network. Comprehensive molecular characterization of clear cell renal cell carcinoma. Nature. 2013;499:43-49.

38. Scelo G, Riazalhosseini Y, Greger L, Letourneau L, GonzàlezPorta M, Wozniak MB, et al. Variation in genomic landscape of clear cell renal cell carcinoma across Europe. Nat Commun. 2014;5:5135.

39. Riazalhosseini Y, Lathrop M. Precision medicine from the renal cancer genome. Nat Rev Nephrol. 2016;12:655-66.

40. Mizutani A, Koinuma D, Seimiya H, Miyazono K. The ArkadiaESRP2 axis suppresses tumor progression: analyses in clear-cell renal cell carcinoma. Oncogene. 2016;35:3514-23.

41. Katsura A, Tamura Y, Hokari S, Harada M, Morikawa M, Sakurai T, et al. ZEB1-regulated inflammatory phenotype in breast cancer cells. Mol Oncol. 2017;11:1241-62.

42. Murai F, Koinuma D, Shinozaki-Ushiku A, Fukayama M, Miyazono K, Ehata S. EZH2 promotes progression of small cell lung cancer by suppressing the TGF- $\beta$-Smad-ASCL1 pathway. Cell Discov. 2015;1:15026.

43. Hoshino Y, Katsuno Y, Ehata S, Miyazono K. Autocrine TGF- $\beta$ protects breast cancer cells from apoptosis through reduction of BH3-only protein, Bim. J Biochem. 2011;149:55-65.

44. Nagata M, Goto K, Ehata S, Kobayashi N, Saitoh M, Miyoshi H, et al. Nuclear and cytoplasmic c-Ski differently modulate cellular functions. Genes Cells. 2006;11:1267-80.

45. Naito S, von Eschenbach AC, Giavazzi R, Fidler IJ. Growth and metastasis of tumor cells isolated from a human renal cell carcinoma implanted into different organs of nude mice. Cancer Res. 1986;46:4109-15.

46. Kubota SI, Takahashi K, Nishida J, Morishita Y, Ehata S, Tainaka $\mathrm{K}$, et al. Whole-body profiling of cancer metastasis with singlecell resolution. Cell Rep. 2017;20:236-50.

47. Yokoyama Y, Watanabe T, Tamura Y, Hashizume Y, Miyazono $\mathrm{K}$, Ehata S. Autocrine BMP-4 signaling is a therapeutic target in colorectal cancer. Cancer Res. 2017;77:4026-38.

48. Huang DW, Sherman BT, Lempicki RA. Bioinformatics enrichment tools: paths toward the comprehensive functional analysis of large gene lists. Nucleic Acids Res. 2009;37:1-13.

49. Huang DW, Sherman BT, Lempicki RA. Systematic and integrative analysis of large gene lists using DAVID bioinformatics resources. Nat Protoc. 2009;4:44-57. 